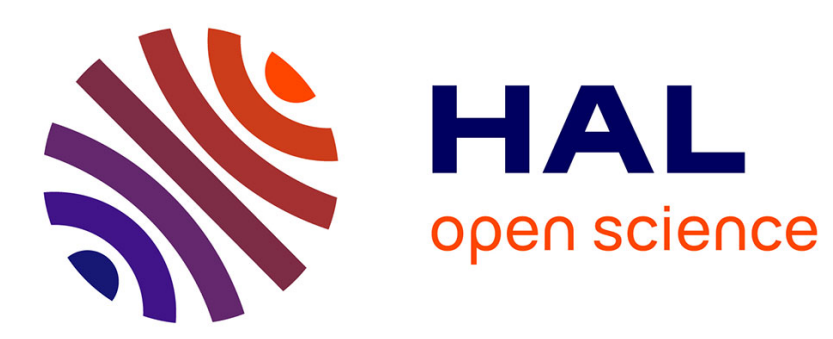

\title{
Efficient computation of minmax tests for fault isolation and their application to structural damage localization
}

Michael Döhler, Laurent Mevel, Falk Hille

\section{To cite this version:}

Michael Döhler, Laurent Mevel, Falk Hille. Efficient computation of minmax tests for fault isolation and their application to structural damage localization. IFAC WC - 19th IFAC World Congress, Aug 2014, Cape Town, South Africa. 10.3182/20140824-6-ZA-1003.00881 . hal-00976009

\section{HAL Id: hal-00976009 \\ https://hal.inria.fr/hal-00976009}

Submitted on 3 Sep 2014

HAL is a multi-disciplinary open access archive for the deposit and dissemination of scientific research documents, whether they are published or not. The documents may come from teaching and research institutions in France or abroad, or from public or private research centers.
L'archive ouverte pluridisciplinaire HAL, est destinée au dépôt et à la diffusion de documents scientifiques de niveau recherche, publiés ou non, émanant des établissements d'enseignement et de recherche français ou étrangers, des laboratoires publics ou privés. 


\title{
Efficient Computation of Minmax Tests for Fault Isolation and Their Application to Structural Damage Localization *
}

\author{
Michael Döhler* Laurent Mevel ${ }^{* *}$ Falk Hille* \\ * BAM Federal Institute for Materials Research and Testing, Safety of \\ Structures Department, 12200 Berlin, Germany (e-mail: \\ michael.doehler@bam.de, falk.hille@bam.de). \\ ** Inria, Campus de Beaulieu, 35042 Rennes, France (e-mail: \\ laurent.mevel@inria.fr).
}

\begin{abstract}
Fault detection and isolation can be handled by many different approaches. This paper builds upon a hypothesis test that checks whether the mean of a Gaussian random vector has become non-zero in the faulty state, based on a chi2 test. For fault isolation, it has to be decided which components in the parameter set of the Gaussian vector have changed, which is done by variants of the chi2 hypothesis test using the so-called sensitivity and minmax approaches. While only the sensitivity of the tested parameter component is taken into account in the sensitivity approach, the sensitivities of all parameters are used in the minmax approach, leading to better statistical properties at the expense of an increased computational burden. The computation of the respective test variable in the minmax test is cumbersome and may be illconditioned especially for large parameter sets, asking hence for a careful numerical evaluation. Furthermore, the fault isolation procedure requires the repetitive calculation of the test variable for each of the parameter components that are tested for a change, which may be a significant computational burden. In this paper, dealing with the minmax problem, we propose a new efficient computation for the test variables, which is based on a simultaneous QR decomposition for all parameters. Based on this scheme, we propose an efficient test computation for a large parameter set, leading to a decrease in the numerical complexity by one order of magnitude in the total number of parameters. Finally, we show how the minmax test is useful for structural damage localization, where an asymptotically Gaussian residual vector is computed from outputonly vibration data of a mechanical or a civil structure.
\end{abstract}

Keywords: Fault isolation, residual evaluation, statistical tests, numerical computation, mechanical systems.

\section{INTRODUCTION}

Fault detection and isolation (FDI) are of practical importance in many branches of automatic control and have found a strong interest in connected areas [Hwang et al., 2010]. In structural engineering, they have found an application for vibration monitoring of civil, aeronautical or mechanical structures in order to detect damages (fault detection) and to locate damages (fault isolation) [Basseville et al., 2000, 2004, Döhler and Mevel, 2013]. For such applications, FDI is based on a Gaussian residual vector with zero mean in the reference state and non-zero mean in the faulty state. Based on a parameterization of the investigated system, hypothesis tests are performed on the Gaussian vector for FDI [Basseville and Nikiforov, 1993, Basseville, 1997].

For fault isolation, it has to be decided which components in the parameter set of the Gaussian vector have changed, which can be done by variants of the hypothesis test using the so-called sensitivity or minmax approaches. While only

* This work was supported by the European project FP7-PEOPLE2009-IAPP 251515 ISMS. the sensitivity of the tested parameter component is taken into account in the sensitivity approach, the sensitivities of all parameters are used in the minmax approach, leading to better statistical properties at the expense of an increased computational burden. Moreover, the estimated covariance of the Gaussian vector may be badly conditioned due to noise and insufficient data, which leads to an ill-conditioned computation of the respective test variable in the FDI tests. In Zhang and Basseville [2003], a numerically robust computation of both statistical tests was presented. The robust computation is based on the pertinent use of the QR decomposition applied to the sensitivity and covariance parameters of the Gaussian vector. While significantly improving the computation of these tests and making them possible for practical applications, the computations can further be optimized. Furthermore, fault isolation often requires the repetitive calculation of the test variable for the different parameters that are tested for a change, in order to identify which of these parameters actually have changed, which may represent a significant computational burden. In this paper, we improve the numerical efficiency of the computation of the test variable 
in the minmax test from Zhang and Basseville [2003] by reducing the number of necessary $\mathrm{QR}$ decompositions from three to one. Based on this development, we propose an efficient test computation for large parameter sets, leading to a decrease in the numerical complexity by one order of magnitude in the total number of parameters.

For structural vibration monitoring, output-only measurements are taken from a linear system subject to state and measurement noise [Juang, 1994]. Subspace methods have shown excellent properties for the treatment of such data [Van Overschee and De Moor, 1996, Peeters and De Roeck, 1999, Basseville et al., 2007, Döhler and Mevel, 2012]. An asymptotically Gaussian subspace-based residual vector for FDI has been proposed in Basseville et al. [2000], which is used for damage localization in [Basseville et al., 2004, Balmès et al., 2008], corresponding to fault isolation using a parameterization that is linked to local structural properties. However, in these works only the sensitivity test was used, whereas the minmax test has more appropriate properties. In this paper, we extend the previous results on structural damage localization with the minmax test. In this context, both the numerical robust computation and the efficient test computation for many parameters are of importance, the former due to noisy data and the latter since parameter sets can be huge in this application.

This paper is organized as follows. In Section 2, the sensitivity and the minmax tests are recalled. In Section 3 , the numerically robust computation of these tests is recalled from [Zhang and Basseville, 2003] and a more efficient and fast approach for multiple parameter tests is derived. These tests are shown in the context of structural damage localization in Section 4. In Section 5, an application on a simulated flexural beam is shown, where the minmax test outperforms the sensitivity test, before stating some concluding remarks in Section 6 .

\section{BASIC FAULT ISOLATION TESTS}

We consider the problem of isolating changes in a parameter $\theta \in \mathbb{R}^{l}$ of a Gaussian residual vector $\zeta=\zeta(\theta) \in \mathbb{R}^{h}$ in a faulty state, following the distribution

$$
\zeta \sim \begin{cases}\mathcal{N}(0, \Sigma) & \text { under } \mathbf{H}_{0} \\ \mathcal{N}(\mathcal{J} \delta \theta, \Sigma) & \text { under } \mathbf{H}_{1}\end{cases}
$$

where the null hypothesis $\mathbf{H}_{0}$ corresponds to a reference state and $\mathbf{H}_{1}$ to a faulty state. The vector $\delta \theta \in \mathbb{R}^{l}$ denotes the parameter change, the matrix $\mathcal{J} \in \mathbb{R}^{h \times l}$ with full column rank denotes the sensitivity of the residual vector, and the positive definite matrix $\Sigma \in \mathbb{R}^{h \times h}$ is its covariance. Many methods have been proposed in the literature for fault detection (decide if there is a change in $\theta$ ) and isolation (decide which elements of vector $\theta$ have changed) by the means of such a Gaussian residual vector $\zeta$, see e.g. Basseville and Nikiforov [1993], Basseville [1997].

For fault isolation, different partitions of the vector $\delta \theta$ into two subvectors are considered, where each time a decision is made if the first subvector is zero or not. Without loss of generality, let this partition be

$$
\delta \theta=\left[\begin{array}{l}
\delta \theta_{a} \\
\delta \theta_{b}
\end{array}\right]
$$

Let $F=\mathcal{J}^{T} \Sigma^{-1} \mathcal{J}$ be the Fisher information matrix of the parameter $\theta$ contained in vector $\zeta$, and let $\mathcal{J}$ and $F$ be partitioned accordingly as

$$
\mathcal{J}=\left[\begin{array}{ll}
\mathcal{J}_{a} & \mathcal{J}_{b}
\end{array}\right], F=\left[\begin{array}{cc}
F_{a a} & F_{a b} \\
F_{b a} & F_{b b}
\end{array}\right]=\left[\begin{array}{cc}
\mathcal{J}_{a}^{T} \Sigma^{-1} \mathcal{J}_{a} & \mathcal{J}_{a}^{T} \Sigma^{-1} \mathcal{J}_{b} \\
\mathcal{J}_{b}^{T} \Sigma^{-1} \mathcal{J}_{a} & \mathcal{J}_{b}^{T} \Sigma^{-1} \mathcal{J}_{b}
\end{array}\right]
$$

In the following, two isolation tests are recalled as stated in Zhang and Basseville [2003], where a decision is made between $\delta \theta_{a}=0$ and $\delta \theta_{a} \neq 0$ in partition (2). Both tests will be applied to a subspace-based residual vector with property (1) to perform fault isolation in the context of vibration-based structural damage localization [Basseville et al., 2004, Balmès et al., 2008] in Section 4.

\subsection{Sensitivity Test}

The simplest possibility for testing changes $\delta \theta_{a}$ is to assume $\delta \theta_{b}=0$ and thus $\zeta \sim \mathcal{N}\left(\mathcal{J}_{a} \delta \theta_{a}, \Sigma\right)$. The corresponding Generalized Likelihood Ratio (GLR) test writes as [Basseville, 1997]

$$
t_{\text {sens }}=\zeta^{T} \Sigma^{-1} \mathcal{J}_{a}\left(\mathcal{J}_{a}^{T} \Sigma^{-1} \mathcal{J}_{a}\right)^{-1} \mathcal{J}_{a}^{T} \Sigma^{-1} \zeta,
$$

which is called sensitivity test. The test variable $t_{\text {sens }}$ is $\chi^{2}$-distributed with $\operatorname{dim}\left(\theta_{a}\right)$ degrees of freedom and noncentrality parameter $\delta \theta_{a}^{T} F_{a a} \delta \theta_{a}$, if $\delta \theta_{b}=0$ is actually true. For a decision, the test variable is compared to a threshold.

\subsection{Minmax Test}

Instead of assuming $\delta \theta_{b}=0$, the variable $\delta \theta_{b}$ is replaced by its least favorable value for a decision about $\delta \theta_{a}$, as follows. Define the partial residuals as

$$
\begin{gathered}
\zeta_{a} \stackrel{\text { def }}{=} \mathcal{J}_{a}^{T} \Sigma^{-1} \zeta, \\
\zeta_{b} \stackrel{\text { def }}{=} \mathcal{J}_{b}^{T} \Sigma^{-1} \zeta,
\end{gathered}
$$

the robust residual as [Basseville, 1997]

$$
\zeta_{a}^{*} \stackrel{\text { def }}{=} \zeta_{a}-F_{a b} F_{b b}^{-1} \zeta_{b}
$$

and

$$
F_{a}^{*} \stackrel{\text { def }}{=} F_{a a}-F_{a b} F_{b b}^{-1} F_{b a} .
$$

Then, the mean of the robust residual $\zeta_{a}^{*}$ is sensitive to changes $\delta \theta_{a}$, but blind to $\delta \theta_{b}$, and it holds

$$
\zeta_{a}^{*} \sim \mathcal{N}\left(F_{a}^{*} \delta \theta_{a}, F_{a}^{*}\right)
$$

The corresponding GLR test writes as

$$
t_{\mathrm{mm}}=\zeta_{a}^{* T} F_{a}^{*-1} \zeta_{a}^{*}
$$

which is called minmax test. The test variable $t_{\mathrm{mm}}$ is $\chi^{2}$-distributed with $\operatorname{dim}\left(\theta_{a}\right)$ degrees of freedom and noncentrality parameter $\delta \theta_{a}^{T} F_{a}^{*} \delta \theta_{a}$, independently of $\delta \theta_{b}$. For a decision, the test variable is compared to a threshold. Note that the invertibility of all matrices in the computation is guaranteed, since $\mathcal{J}$ is assumed to have full column rank and $\Sigma$ is positive definite.

\section{NUMERICALLY EFFICIENT COMPUTATION}

Both the sensitivity and the minmax test require a number of matrix inversions, which may be numerically critical due to possible ill-conditioning of the covariance matrix $\Sigma$. In [Zhang and Basseville, 2003] numerically more robust computations of both tests were suggested, making use of the decomposition

$$
\Sigma^{-1}=\left(\Sigma^{-1 / 2}\right)^{T} \Sigma^{-1 / 2}
$$


and a number of QR decompositions. Note that an efficient computation of $\Sigma^{-1 / 2}$ is detailed in [Döhler and Mevel, 2011, Döhler et al., 2014], and let $n_{r} \times h$ be the dimensions of $\Sigma^{-1 / 2}$ that may be different from each other.

After recalling the robust computations from [Zhang and Basseville, 2003] for completeness, we improve the computation of the minmax test in this section. While the computation in [Zhang and Basseville, 2003] needs three QR decompositions, we suggest a more efficient computation that just needs one simultaneous QR decomposition. Based on this development, we furthermore propose a fast computation for multiple minmax tests, where different subvectors of $\delta \theta$ are tested.

\subsection{Results from [Zhang and Basseville, 2003]}

Sensitivity Test Using the thin QR decomposition [Golub and Van Loan, 1996]

$$
\Sigma^{-1 / 2} \mathcal{J}_{a}=Q R
$$

the sensitivity test in (4) writes as

$$
t_{\text {sens }}=\alpha^{T} \alpha, \text { where } \alpha=Q^{T} \Sigma^{-1 / 2} \zeta .
$$

Here, the number of numerically critical matrix inversions and multiplications is limited to a minimum compared to a direct computation in (4).

\section{Minmax Test Using the thin QR decompositions}

$$
\begin{aligned}
\Sigma^{-1 / 2} \mathcal{J}_{a} & =\tilde{Q}_{a} \tilde{R}_{a}, \quad \Sigma^{-1 / 2} \mathcal{J}_{b}=\tilde{Q}_{b} \tilde{R}_{b}, \\
\left(I-\tilde{Q}_{b} \tilde{Q}_{b}^{T}\right) \tilde{Q}_{a} & =\tilde{Q}_{c} \tilde{R}_{c},
\end{aligned}
$$

the minmax test in (8) writes as

$$
t_{\mathrm{mm}}=\tilde{\beta}^{T} \tilde{\beta}, \quad \text { where } \tilde{\beta}=\tilde{Q}_{c}^{T} \Sigma^{-1 / 2} \zeta .
$$

Again, the number of numerically critical operations is strongly reduced.

\subsection{Minmax Test Revisited}

In the following theorem, a more direct and more efficient computation than in the previous section is shown.

Theorem 1. Let the thin QR decomposition of

$$
\Sigma^{-1 / 2}\left[\begin{array}{ll}
\mathcal{J}_{b} & \mathcal{J}_{a}
\end{array}\right]=\left[\begin{array}{ll}
Q_{b} & Q_{a}
\end{array}\right]\left[\begin{array}{cc}
R_{b b} & R_{b a} \\
0 & R_{a a}
\end{array}\right]
$$

be given and partitioned accordingly. Then, the minmax test in (8) writes as

$$
t_{\mathrm{mm}}=\beta^{T} \beta, \quad \text { where } \beta=Q_{a}^{T} \Sigma^{-1 / 2} \zeta .
$$

Proof. From the QR decomposition (9) it follows

$$
\begin{aligned}
& \Sigma^{-1 / 2} \mathcal{J}_{a}=Q_{b} R_{b a}+Q_{a} R_{a a} \\
& \Sigma^{-1 / 2} \mathcal{J}_{b}=Q_{b} R_{b b}
\end{aligned}
$$

where $Q_{a}^{T} Q_{a}=I, Q_{b}^{T} Q_{b}=I$ and $Q_{b}^{T} Q_{a}=0$, and thus in (3)

$$
F=\left[\begin{array}{cc}
F_{a a} & F_{a b} \\
F_{b a} & F_{b b}
\end{array}\right]=\left[\begin{array}{cc}
R_{b a}^{T} R_{b a}+R_{a a}^{T} R_{a a} & R_{b a}^{T} R_{b b} \\
R_{b b}^{T} R_{b a} & R_{b b}^{T} R_{b b}
\end{array}\right] .
$$

The partial residuals in (5a) and (5b) write as

$$
\begin{aligned}
\zeta_{a} & =R_{b a}^{T} Q_{b}^{T} \Sigma^{-1 / 2} \zeta+R_{a a}^{T} Q_{a}^{T} \Sigma^{-1 / 2} \zeta, \\
\zeta_{b} & =R_{b b}^{T} Q_{b}^{T} \Sigma^{-1 / 2} \zeta,
\end{aligned}
$$

and the robust residual in (6) yields

$$
\begin{aligned}
\zeta_{a}^{*}= & \zeta_{a}-R_{b a}^{T} R_{b b}\left(R_{b b}^{T} R_{b b}\right)^{-1} \zeta_{b} \\
= & R_{b a}^{T} Q_{b}^{T} \Sigma^{-1 / 2} \zeta+R_{a a}^{T} Q_{a}^{T} \Sigma^{-1 / 2} \zeta \\
& \quad-R_{b a}^{T} R_{b b}\left(R_{b b}^{T} R_{b b}\right)^{-1} R_{b b}^{T} Q_{b}^{T} \Sigma^{-1 / 2} \zeta \\
= & R_{a a}^{T} Q_{a}^{T} \Sigma^{-1 / 2} \zeta .
\end{aligned}
$$

Similarly, substituting the elements of $F_{a}^{*}$ in (7) with (10) yields

$$
\begin{aligned}
F_{a}^{*} & =\left(R_{b a}^{T} R_{b a}+R_{a a}^{T} R_{a a}\right)-R_{b a}^{T} R_{b b}\left(R_{b b}^{T} R_{b b}\right)^{-1} R_{b b}^{T} R_{b a} \\
& =R_{a a}^{T} R_{a a} .
\end{aligned}
$$

Finally, replacing $\zeta_{a}^{*}$ and $F_{a}^{*}$ in the minmax test (8) yields

$$
\begin{aligned}
t_{\mathrm{mm}} & =\zeta^{T}\left(\Sigma^{-1 / 2}\right)^{T} Q_{a} R_{a a}\left(R_{a a}^{T} R_{a a}\right)^{-1} R_{a a}^{T} Q_{a}^{T} \Sigma^{-1 / 2} \zeta \\
& =\zeta^{T}\left(\Sigma^{-1 / 2}\right)^{T} Q_{a} Q_{a}^{T} \Sigma^{-1 / 2} \zeta,
\end{aligned}
$$

leading to the assertion.

This theorem makes the operation of the minmax test directly visible, when writing it equivalently as $t_{\mathrm{mm}}=\bar{\beta}^{T} \bar{\beta}$ with $\bar{\beta}=Q_{a} Q_{a}^{T} \Sigma^{-1 / 2} \zeta$. Note that $Q_{a} Q_{a}^{T}$ defines an orthogonal projection. So in fact the squared norm of a vector $\bar{\beta}$ is computed, which is the orthogonal projection of the covariance-normalized residual into the subspace of the normalized "change directions" $\Sigma^{-1 / 2} \mathcal{J}_{a}$ that are of interest and that are orthogonal to the normalized change directions $\Sigma^{-1 / 2} \mathcal{J}_{b}$. Like this the projection is blind to changes in the parameters $\theta_{b}$, while only possible changes in parameters $\theta_{a}$ remain in the projected vector. Note that this means in practice that the sensitivities of all parameters in $\mathcal{J}$ need to be sufficiently pairwise distinct, otherwise the effect of a tested parameter in $\delta \theta_{a}$ might be removed by another parameter in the projection. This also corresponds to the necessity of (numerical) invertibility of the $R$ matrix in (9) and thus of $R_{a a}$ and $R_{b b}$ in the proof of Theorem 1, which is in theory given through the full column rank of $\mathcal{J}$ and the positive definiteness of $\Sigma$. However, if these matrices are too badly conditioned because some parameters in $\theta$ are too close, the minmax test might not perform as expected, which is even more the case in its original formulation in Section 2.2.

Compared to the computation proposed in [Zhang and Basseville, 2003], Theorem 1 provides a more direct approach, where only one instead of three QR decompositions and only about half the numerical operations are necessary. Moreover, a fast iterative update of the QR decomposition for testing multiple subsets becomes possible thanks to the direct computation in the new approach, as described in the following section.

\subsection{Minmax Tests on Multiple Parameter Subsets}

When not only one subset $\theta_{a}$ of parameter $\theta$ is tested for a change, but many different subsets, the QR decomposition in (9) is necessary for each tested parameter subset, which is a significant computational burden if the number of parameters $l$ is high.

Without loss of generality, we assume $\theta_{a}$ to be onedimensional in the following for simplicity of notation. Denote the elements of parameter $\theta=\left[\begin{array}{llll}\theta^{1} & \theta^{2} \ldots \theta^{l}\end{array}\right]^{T}$. 
Thus, when testing parameter $\theta^{i}$ for a change, denote $\theta_{a}$ and $\theta_{b}$ from the previous section as

$$
\begin{aligned}
\theta_{a}^{i} & =\theta^{i}, \\
\theta_{b}^{i} & =\left[\begin{array}{llllll}
\theta^{i+1} & \ldots & \theta^{l} & \theta^{1} & \ldots & \theta^{i-1}
\end{array}\right]^{T},
\end{aligned}
$$

and the corresponding sensitivity matrices as $\mathcal{J}_{a}^{i}$ and $\mathcal{J}_{b}^{i}$, respectively.

Instead of computing the QR decomposition in (9) for each parameter $i$, the fact can be used that the columns of the sensitivity matrix $\mathcal{J}$ are the same all the time, but only differently ordered in $\left[\begin{array}{ll}\mathcal{J}_{b}^{i} & \mathcal{J}_{a}^{i}\end{array}\right]$. In the following, an efficient iterative computation of the QR decomposition for the different parameters is derived.

Assume that the thin QR decomposition is given for parameter $i$ in (9) as

$$
\mathcal{K}^{i} \stackrel{\text { def }}{=} \Sigma^{-1 / 2}\left[\begin{array}{ll}
\mathcal{J}_{b}^{i} & \mathcal{J}_{a}^{i}
\end{array}\right]=Q^{i} R^{i},
$$

where $\mathcal{K}^{i} \in \mathbb{R}^{n_{r} \times l}$. At this stage, the vector $Q_{a}^{i}$ in $Q^{i}=$ $\left[\begin{array}{ll}Q_{b}^{i} & Q_{a}^{i}\end{array}\right]$ is used for the minmax test of parameter $\theta_{a}^{i}$ in Theorem 1 in the previous section.

In the next step, it is convenient to compute the QR decomposition for parameter $i+1$ to perform the respective minmax test, where the decomposition

$$
\mathcal{K}^{i+1}=\Sigma^{-1 / 2}\left[\mathcal{J}_{b}^{i+1} \mathcal{J}_{a}^{i+1}\right]=Q^{i+1} R^{i+1} .
$$

is required. It can be obtained efficiently as follows. The matrices $\mathcal{K}^{i}$ and $\mathcal{K}^{i+1}$ are related by a permutation of one column due to the definition in (11a)-(11b): partitioning $\mathcal{K}^{i}=\left[\begin{array}{ll}k_{1} & K_{2}\end{array}\right]$ with $k_{1}$ being the first column leads to $\mathcal{K}^{i+1}=\left[\begin{array}{ll}K_{2} & k_{1}\end{array}\right]$. Partitioning $R^{i}=\left[\begin{array}{ll}r_{1} & R_{2}\end{array}\right]$ analogously, it follows $\mathcal{K}^{i}=\left[\begin{array}{lll}Q^{i} r_{1} & Q^{i} R_{2}\end{array}\right]$ from (12) and thus

$$
\mathcal{K}^{i+1}=Q^{i}\left[\begin{array}{ll}
R_{2} & r_{1}
\end{array}\right] \text {. }
$$

The matrix $\left[R_{2} r_{1}\right]$ is upper Hessenberg due to the upper triangular structure of $R^{i}=\left[\begin{array}{ll}r_{1} & R_{2}\end{array}\right]$. Hence, there are Givens rotations $G_{1}, \ldots, G_{l-1}$, such that the product $G_{l-1}^{T} \cdots G_{1}^{T}\left[R_{2} r_{1}\right]$ is upper triangular [Golub and Van Loan, 1996], and the QR decomposition of the next iteration in (13) can be obtained efficiently from

$$
Q^{i+1}=Q^{i} G_{1} \cdots G_{l-1}, \quad R^{i+1}=G_{l-1}^{T} \cdots G_{1}^{T}\left[R_{2} r_{1}\right] .
$$

This update for $Q^{i+1}$ and $R^{i+1}$ takes only $6 n_{r} l$ flops and $3 l^{2}$ flops, respectively, compared to $4 n_{r} l^{2}-\frac{4}{3} l^{3}$ flops for the direct computation of the thin QR decomposition [Golub and Van Loan, 1996].

The QR decomposition is the dominant operation in the computation of the minmax test, and it holds $n_{r} \geq l$. Testing all parameters $i=1, \ldots, l$ with the minmax test requires thus $\mathcal{O}\left(n_{r} l^{2}\right)$ flops with the iterative scheme in (14), compared to $\mathcal{O}\left(n_{r} l^{3}\right)$ flops for the direct computation.

\section{VIBRATION-BASED STRUCTURAL DAMAGE LOCALIZATION}

Based on the asymptotic local approach for change detection [Benveniste et al., 1987], a subspace-based residual vector has been proposed in Basseville et al. [2000] that asymptotically satisfies relation (1). It found an interesting application in vibration monitoring of civil, aeronautical or mechanical structures for damage detection and localization [Basseville et al., 2004, Balmès et al., 2008]. For the localization problem, a structural parameterization vector $\theta$ in connection with a finite element model (FEM) of the structure is used, whose components refer to local structural properties. Damage localization thus corresponds to isolating the changed components of $\theta$ by means of the residual vector.

In this section, the subspace-based damage localization method is recalled from [Basseville et al., 2004, Balmès et al., 2008], where, however, only the sensitivity test was used for fault isolation. We extend these results with the minmax test in this section.

\subsection{The Residual Vector}

The behavior of linear time-invariant dynamical structures subject to unknown ambient excitation can be described by system

$$
\mathcal{M} \ddot{\mathcal{X}}(t)+\mathcal{C} \dot{\mathcal{X}}(t)+\mathcal{K} \mathcal{X}(t)=v(t)
$$

where $t$ denotes continuous time; $\mathcal{M}, \mathcal{C}, \mathcal{K} \in \mathbb{R}^{m \times m}$ are mass, damping, and stiffness matrices, respectively; the (high dimensional) state vector $\mathcal{X}(t) \in \mathbb{R}^{m}$ is the displacement vector of the $m$ degrees of freedom of the structure; and $v(t)$ is the external unmeasured force (noise). Observing system (15) at $r$ sensor positions (e.g. acceleration or displacement sensors) at discrete time instants $t=k \tau$ (with sampling rate $1 / \tau$ ), it can be transformed to an equivalent discrete-time state space system [Juang, 1994]

$$
\left\{\begin{aligned}
x_{k+1} & =A x_{k}+v_{k} \\
y_{k} & =C x_{k}+w_{k}
\end{aligned}\right.
$$

with the states $x_{k} \in \mathbb{R}^{n}$, the measured outputs $y_{k} \in \mathbb{R}^{r}$, the state transition matrix $A \in \mathbb{R}^{n \times n}$ and the observation matrix $C \in \mathbb{R}^{r \times n}$, where $n=2 m$ is the model order and $r$ the number of outputs. The state noise $v_{k}$ and output noise $w_{k}$ are unmeasured and assumed to be Gaussian, zero-mean, white. The eigenvalues and eigenvectors at the observed degrees of freedom of model (15) constitute a canonical parameterization that can be equivalently obtained from the eigenstructure of model (16) [Juang, 1994]. The modal parameters (frequencies, damping ratios and mode shapes) are deduced from the eigenstructure, and their changes indicate damages (faults). While fault detection can thus be built on changes in the eigenstructure, fault isolation for damage localization is built on a parameterization containing local structural parameters later on.

First, a subspace-based residual vector is defined as follows [Basseville et al., 2000]. Let $G=\mathbf{E}\left(x_{k+1} y_{k}^{T}\right)$ be the cross-covariance between the states and the outputs, let $R_{i}=\mathbf{E}\left(y_{k} y_{k-i}^{T}\right)=C A^{i-1} G(i \geq 1)$ be the theoretic output covariances and let

$$
\mathcal{H}_{p+1, q} \stackrel{\text { def }}{=}\left[\begin{array}{cccc}
R_{1} & R_{2} & \ldots & R_{q} \\
R_{2} & R_{3} & \ldots & R_{q+1} \\
\vdots & \vdots & \ddots & \vdots \\
R_{p+1} & R_{p+2} & \ldots & R_{p+q}
\end{array}\right] \stackrel{\text { def }}{=} \operatorname{Hank}\left(R_{i}\right)
$$

be the theoretic block Hankel matrix with the well-known factorization property

$$
\mathcal{H}_{p+1, q}=\mathcal{O}_{p+1} \mathcal{C}_{q}
$$

into observability and controllability matrices 


$$
\mathcal{O}_{p+1}=\left[\begin{array}{c}
C \\
C A \\
\vdots \\
C A^{p}
\end{array}\right], \quad \mathcal{C}_{q}=\left[\begin{array}{llll}
G & A G \ldots & A^{q-1} G
\end{array}\right] .
$$

Parameters $p$ and $q$ are chosen such that $\operatorname{rank}\left(\mathcal{O}_{p}\right)=$ $\operatorname{rank}\left(\mathcal{C}_{q}\right)=n$. In the reference state, the left null space $S$ of observability matrix $\mathcal{O}_{p+1}$ is obtained, such that $S^{T} \mathcal{O}_{p+1}=0$. Then, $S$ defines also a basis of the left null space of $\mathcal{H}_{p+1, q}$ in the reference state because of factorization property (18), and the characteristic property of a system in the reference state writes [Basseville et al., 2000]

$$
S^{T} \mathcal{H}_{p+1, q}=0 .
$$

From the outputs $\left\{y_{k}: k=1, \ldots, N\right\}$, a consistent estimate $\widehat{\mathcal{H}}_{p+1, q}=\operatorname{Hank}\left(\widehat{R}_{i}\right)$ is obtained from the estimated output covariances $\widehat{R}_{i}=1 / N \sum_{k=1}^{N} y_{k} y_{k-i}^{T}$. Based on property (19), the residual vector

$$
\zeta_{N}=\sqrt{N} \operatorname{vec}\left(S^{T} \widehat{\mathcal{H}}_{p+1, q}\right)
$$

is defined, which has the property $\mathbf{E}\left(\zeta_{N}\right)=0$ iff the system is in the reference state.

\subsection{Fault Isolation Tests for Damage Localization}

The evaluation of the residual is possible based on the asymptotic local approach for change detection [Benveniste et al., 1987], assuming the close hypotheses

$$
\begin{array}{ll}
\mathbf{H}_{0}: \theta=\theta_{0} & \text { (reference system) } \\
\mathbf{H}_{1}: \theta=\theta_{0}+\delta \theta / \sqrt{N} & \text { (faulty system) }
\end{array}
$$

where vector $\theta_{0}$ denotes the system parameter in the reference state and vector $\delta \theta$ is unknown but fixed. With this statistical framework, very small changes in the system parameter $\theta$ can be detected if $N$ is large enough. The subspace-based residual $\zeta_{N}$ yields [Basseville et al., 2000]

$$
\zeta_{N} \stackrel{d}{\longrightarrow} \begin{cases}\mathcal{N}(0, \Sigma) & \text { under } \mathbf{H}_{0} \\ \mathcal{N}(\mathcal{J} \delta \theta, \Sigma) & \text { under } \mathbf{H}_{1}\end{cases}
$$

for $N \rightarrow \infty$, where $\mathcal{J}$ and $\Sigma$ are the asymptotic sensitivity and covariance, respectively. The estimation of $\mathcal{J}$ is described in detail in [Basseville et al., 2004, Balmès et al., 2008 for a chosen structural parameterization $\theta$, and the estimation of $\Sigma$ is described in [Döhler and Mevel, 2011], both in the reference state.

Thanks to property (22), both the sensitivity and the minmax test can be applied to residual $\zeta_{N}$ (asymptotically, $N$ large enough) for each of the structural parameters in $\theta=\left[\theta^{1} \ldots \theta^{l}\right]$. A test value for a parameter above a certain threshold corresponds to a damage location in the structure associated with the parameter. In this respect, the preferred test should be the minmax test instead of the sensitivity test used in [Basseville et al., 2004, Balmès et al., 2008], since the condition $\delta \theta_{b}=0$ can never be guaranteed when testing $\delta \theta_{a}=\delta \theta^{i}$ for some parameter $i$. As all parameters of $\theta$ are tested successively, the efficient test computation of Section 3.3 is beneficial especially for large parameterizations.

As noted in Section 3, care must be taken that $\mathcal{J}$ is wellconditioned for the minmax test, i.e. that the structural parameters in $\theta$ are sufficiently distinct. This might require a prior clustering step as already performed in [Basseville et al., 2004, Balmès et al., 2008].

\section{APPLICATION}

The model of a flexural beam structure is considered for the application of the damage localization tests. The model comprises 40 equally sized finite $2 \mathrm{D}$ elastic beam elements (Figure 1) allowing two translational and one rotational displacements at each node. The beam has a fixed support on one end and a movable support on the other end, so the system is statically indeterminate and four of the altogether 123 degrees of freedom of the model are restricted. The beam has invariable cross-sectional and material properties in the undamaged state. The mass is distributed and defined such that the first frequency of the system is at $5 \mathrm{~Hz}$ and damping is defined such that all modes have a damping ratio of $2 \%$.

Damage is modeled by a $20 \%$ reduction of Young's modulus for two damage cases, first in element 10, and second both in elements 10 and 18, having a linear impact on the stiffness at the affected elements. The parameter vector $\theta$ is of dimension 40 and consists of Young's modulus at each of the 40 elements.

For both the reference and the damaged states, a data set with $N=50000$ acceleration samples at ten sensor positions was generated at a sampling frequency of $500 \mathrm{~Hz}$ from white noise excitation. $5 \%$ white noise were added on the generated outputs as measurement noise. All parameters of the localization tests $(S, \mathcal{J}, \Sigma)$ were estimated based on the data set in the reference state and information from the FEM using only the first eight modes. In the damaged state, both the sensitivity and the minmax tests with their efficient numerical computation from Section 3 were applied to the data sets of the two fault scenarios. No comparison was made to the naive computation of the test variables (Sections 2.1 and 2.2), since the ineffectiveness of this computation was already shown in [Zhang and Basseville, 2003].

The respective test values were computed for each of the 40 structural elements and are shown in Figures 2 and 3. In both fault scenarios, the damaged elements have the highest test values, thus correctly indicating the damage locations. However, the test values corresponding to the undamaged elements in the sensitivity test are much higher (in relative terms) than in the minmax test. This behavior was expected since the sensitivity test value for some parameter may also be influenced by a change in another parameter. Thus, the minmax test indeed offers a better "contrast" for fault isolation in our application on damage localization and should be the statistical test of choice for this application.

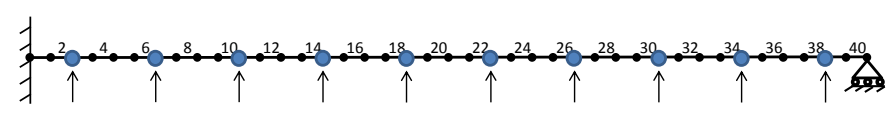

Fig. 1. Flexural beam model with element numbers and ten sensors. 

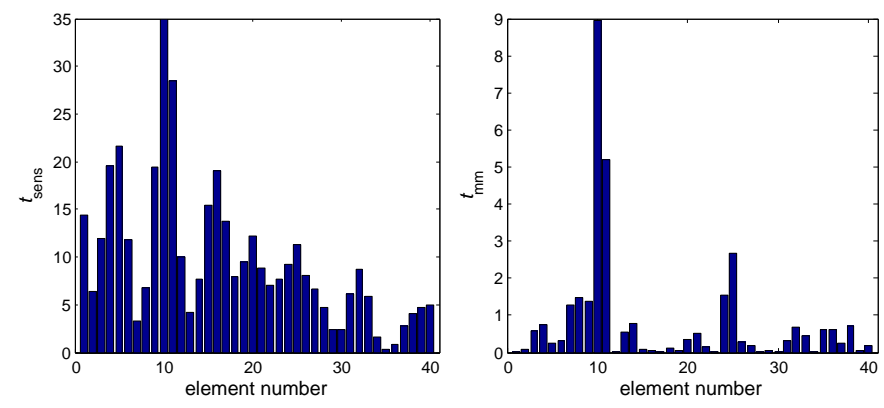

Fig. 2. Sensitivity tests (left) and minmax tests (right) for damage at element 10 .
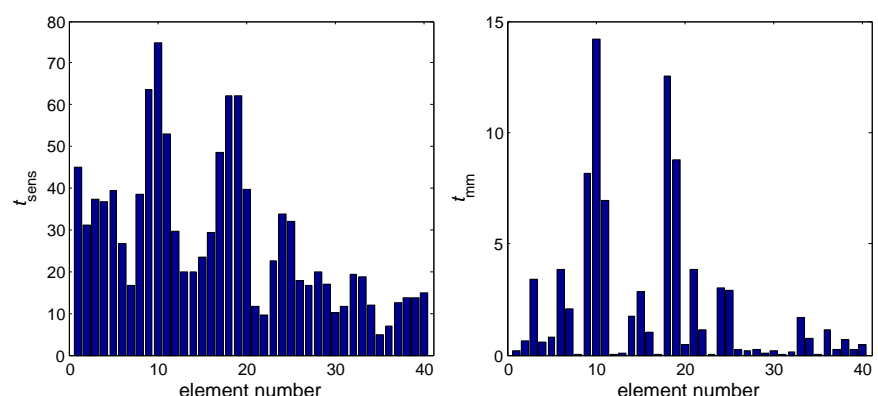

Fig. 3. Sensitivity tests (left) and minmax tests (right) for damage at elements 10 and 18.

\section{CONCLUSIONS}

In this paper, two statistical tests for fault isolation based on a Gaussian residual vector were recalled. A new, numerically more efficient computation for the minmax test was derived in Theorem 1 together with an associated fast computation when testing the different parameters of a large parameter set in Section 3.3, which are the main theoretical results of this paper. An application of this test was shown for vibration-based structural damage localization, where changes in local parameters of a structure indicate damage. In this setting, the minmax test proved to be more effective than the previously used sensitivity test and seems to be promising for applications in structural health monitoring.

\section{REFERENCES}

É. Balmès, M. Basseville, L. Mevel, H. Nasser, and W. Zhou. Statistical model-based damage localization: a combined subspace-based and substructuring approach. Structural Control and Health Monitoring, 15(6):857$875,2008$.

M. Basseville. Information criteria for residual generation and fault detection and isolation. Automatica, 33(5): 783-803, 1997.

M. Basseville and I. Nikiforov. Detection of Abrupt Changes - Theory and Applications. Information and System Sciences Series. Prentice Hall, Englewood Cliffs, NJ, 1993.

M. Basseville, M. Abdelghani, and A. Benveniste. Subspace-based fault detection algorithms for vibration monitoring. Automatica, 36(1):101-109, 2000.
M. Basseville, L. Mevel, and M. Goursat. Statistical model-based damage detection and localization: subspace-based residuals and damage-to-noise sensitivity ratios. Journal of Sound and Vibration, 275(3):769$794,2004$.

M. Basseville, A. Benveniste, M. Goursat, and L. Mevel. Subspace-based algorithms for structural identification, damage detection, and sensor data fusion. EURASIP Journal on Applied Signal Processing, (ID 69136), 2007.

A. Benveniste, M. Basseville, and G.V. Moustakides. The asymptotic local approach to change detection and model validation. IEEE Transactions on Automatic Control, 32(7):583-592, 1987.

M. Döhler and L. Mevel. Robust subspace based fault detection. In Proc. 18th IFAC World Congress, Milan, Italy, 2011.

M. Döhler and L. Mevel. Fast multi-order computation of system matrices in subspace-based system identification. Control Engineering Practice, 20(9):882-894, 2012.

M. Döhler and L. Mevel. Subspace-based fault detection robust to changes in the noise covariances. Automatica, 49(9):2734-2743, 2013.

M. Döhler, L. Mevel, and F. Hille. Subspace-based damage detection under changes in the ambient excitation statistics. Mechanical Systems and Signal Processing, 45 (1):207-224, 2014.

G.H. Golub and C.F. Van Loan. Matrix computations. Johns Hopkins University Press, 3rd edition, 1996.

I. Hwang, S. Kim, Y. Kim, and C. E. Seah. A survey of fault detection, isolation, and reconfiguration methods. IEEE Transactions on Control Systems Technology, 18 (3):636-653, 2010.

J.-N. Juang. Applied system identification. Prentice Hall, Englewood Cliffs, NJ, USA, 1994.

B. Peeters and G. De Roeck. Reference-based stochastic subspace identification for output-only modal analysis. Mechanical Systems and Signal Processing, 13(6):855878, 1999.

P. Van Overschee and B. De Moor. Subspace Identification for Linear Systems: Theory, Implementation, Applications. Kluwer, 1996.

Q. Zhang and M. Basseville. Advanced numerical computation of $\chi^{2}$-tests for fault detection and isolation. In 5th Symp. Fault Detection, Supervision and Safety for Technical Processes (SAFEPROCESS), pages 211-216, Washington, USA, 2003. 